MS07-03

\section{Molecular mechanism of the TP901-1 lysogeny switch}

Leila Lo Leggio ${ }^{1}$, Kim K. Rasmussen ${ }^{1}$, Anders K. Varming ${ }^{1}$, Kristian E.H. Frandsen ${ }^{1}$, Peter W. Thusltrup ${ }^{1}$, Mogens Kilstrup ${ }^{2}$, Karin Hammer $^{2}$, Malene R. Jensen ${ }^{3}$

1. Department of Chemistry, University of Copenhagen, Copenhagen, Denmark

2. DTU Bioengineering, Technical University of Denmark, Kgs. Lyngby, Denmark

3. Univ. Grenoble Alpes, CNRS, CEA, IBS, Grenoble, France email: leila@chem.ku.dk

Bacteriophages are bacterial viruses which can be classified as lytic (virulent) or lysogenic (temperate). Lytic bacteriophages synthesize new progeny directly after infection, whereas lysogenic phages can enter the alternative lysogenic life cycle, where the phage DNA integrates in the bacterium's genome until prompted to switch to the lytic cycle by an external signal. The potential of phages in the fight against antibiotic resistance and increasing understanding of their roles in bacterial pathogenicity have recently rekindled research interest in these diverse and extremely widespread entities.Lysogeny switches have been some of the first models of genetic regulation, but despite superficial similarities, the molecular basis of different switches are rather different. In the dairy industry, phages of lactic acid bacteria are extremely detrimental. Within the last ten years, we have studied the structural biology of the Lactococcus lactis TP901-1 lysogeny switch through an integrative structural biology approach combining biochemistry, crystallography, NMR and CD spectroscopy and small angle scattering. At the DNA level the lysogeny switch of TP901-1 consists of some operator sites and the two genes $c I$ and mor, under the control of two divergent promoters, and coding for the two main regulatory proteins. The repressor CI, alone sufficient to establish lysogeny, consists of an N-terminal DNA binding domain (NTD) of the HTH type (1) connected by a flexible linker (2) to a helical hooks dimerization region $\left(\mathrm{CTD}_{1}\right)(3)$ and an additional helical region which mediates hexamerization $\left(\mathrm{CTD}_{2}\right)$. A dimeric truncated form binds to each of three palindromic operator sites $\left(\mathrm{O}_{R}, \mathrm{O}_{\mathrm{L}}\right.$ and $\left.\mathrm{O}_{\mathrm{D}}\right)$, by binding to adjacent half-sites (3), while the hexamer is thought to bind simultaneously to all three operators, repressing the lytic life cycle promoter $\left(\mathrm{P}_{\mathrm{L}}\right)$. We are pursuing the structure of full-length CI/DNA complex by small angle neutron scattering (SANS). The anti-repressor MOR has previously been suggested to bind to CI, forming a complex, which binds to a still unknown DNA sequence in order to repress the lysogenic life cycle promoter $\left(\mathrm{P}_{\mathrm{R}}\right)$. We recently determined the structure of MOR and its complex with the NTD region of CI, and are further investigating is binding to a composite DNA binding site by biochemical methods and SANS, to give us a full understanding of the function of this simple but efficient switch. As an additional perspective, the interaction of MOR with CI-NTD in TP901-1 is expected to be conserved in putative switches of $S$. aureus phages, which are also under investigation.
References:

[1] Frandsen, KH Rasmussen, KK, Jensen, MR, Hammer, K, Pedersen, M, Poulsen, J-CN, Arleth, L and Lo Leggio L (2013) Biochemistry, 52, 6892-6904

[2] Rasmussen, K.K., Frandsen, K.E.H., Boeri Erba, E., Varming, A.K, Pedersen, M., Hammer, K., Kilstrup, M., Thulstrup, P.W., Blackledge, M., Ringkjøbing Jensen, M. and Lo Leggio, L. (2016) Scientific Reports 6, 29574

[3] Rasmussen, K.K., Varming, A.K., Schmidt, S.N., Frandsen, K.E.H., Thulstrup, P.W., Jensen, M.R., Lo Leggio, L. (2018) FEBS Lett., in press

Keywords: Bacteriophage, genetic switch, repressor/ antirepressor 